\title{
Does livestreaming surgical education have the future? Development and participant evaluation of a national COVID-19 livestreaming initiative
}

\author{
Tim M. Feenstra ${ }^{1}$ (1) - L. Peter van Felius ${ }^{2}$. Menno R. Vriens ${ }^{3}$. Laurents P. S. Stassen ${ }^{4}$ Gijs J. D. van Acker ${ }^{5}$. \\ Els J. M. Nieveen van Dijkum ${ }^{1} \cdot$ Marlies P. Schijven ${ }^{1} \cdot$ H. Jaap Bonjer ${ }^{6}$ on behalf of NVvH Livestream Collaborative \\ Group
}

Received: 27 July 2021 / Accepted: 21 November 2021 / Published online: 6 January 2022

(c) The Author(s), under exclusive licence to Springer Science+Business Media, LLC, part of Springer Nature 2021

\begin{abstract}
Backgrounds COVID-19 related reduction of surgical procedures jeopardizes learning on the job of surgical residents. Many educators resorted to digital resources in the search for alternatives. However, these resources are often limited to the extent they offer resident-surgeon interaction like a joint surgical performance does. Here we present a roadmap of livestreaming surgical procedures, and evaluate how surgical livestreams on human cadavers address the unmet educational needs of surgical residents in our Dutch nationwide initiative.

Methods Technical and organizational feasibility, and definition of outcome deliverables for the livestream series and per livestream were essential in livestream development. Faculty selected interventions, lecture contents, and participant preparations. Appropriate location, technical setup, and support were imperative for a stable, high-quality stream with integrated interaction, while maintaining digital privacy. A survey was sent to livestream participants to evaluate each livestream, and allow for constant improvement during the broadcasting of the series. Only surveys which were completed by surgical residents were included in the analysis of this study.

Results Each livestream attracted 139-347 unique viewers and a total of 307 surveys were completed by participants (response rate of $23-38 \%$ per livestream). Sixty percent of surveys $(n=185)$ were completed by surgical residents. Livestreams were highly valued (appreciation $7.7 \pm 1.1$ and recommendation $8.6 \pm 1.1$ ), especially the live procedures combined with interaction and theoretical backgrounds. Criticized were technical difficulties and timing of the livestreams between 5 and $7 \mathrm{pm}$, which interfered with clinical duties.

Conclusion Livestreaming surgical procedures on human cadavers is a valid and valued solution to augment resident education. Digital privacy and a stable, high-quality interactive stream are essential, as are appropriate moderation and relevant lectures. While livestreaming cannot replace hands-on training in the operating room, it enables surgeon-resident interaction which is key in education — and missed in pre-recorded surgical procedures which are currently available online.
\end{abstract}

Keywords Surgical training $\cdot$ Training $\cdot$ Surgery $\cdot$ Digital $\cdot$ Video $\cdot$ COVID-19

Tim M. Feenstra

tm.feenstra@amsterdamumc.nl

1 Department of Surgery, Amsterdam Gastroenterology and Metabolism, Amsterdam UMC, University of Amsterdam, Meibergdreef 9, 1105 AZ Amsterdam, The Netherlands

2 Amsterdam Skills Centre, Amsterdam, The Netherlands

3 Department of Surgery, University Medical Center Utrecht, Utrecht, The Netherlands
4 Department of Surgery, Maastricht University Medical Center, Maastricht, The Netherlands

5 Department of Surgery, Haaglanden Medical Centre, Den Haag, Zuid-Holland, The Netherlands

6 Department of Surgery, Cancer Centre Amsterdam, Amsterdam UMC, Amsterdam, The Netherlands 
The COVID-19 pandemic has presented significant challenges to the medical community, and surgical practice needed to adjust to the new reality. Elective surgery was scaled down or postponed due to lack of personal protective equipment (PPE) and to enable sufficient capacity for COVID-19 patients on wards and intensive care units (ICUs), and surgical residents were relocated to COVID-19 units to assist in the care on the ward [1]. Consequently, training opportunities offered by clinical activities, especially by direct involvement in surgical procedures have been significantly reduced, with a negative impact on the residents' learning progress $[2,3]$.

Surgical educators were challenged to provide alternatives for surgical training without increasing stress on hospital capacity, and while maintaining social and physical distance. As a result, many educators have resorted to digital education to augment traditional curricula. A preCOVID-19 study from January 2020 concluded that surgical residents use and appreciate these digital resources [4]. Most platforms provide videos, lectures, tips \& techniques, and e-learnings - but none of them offer the opportunity to virtually attend the surgery and interact with the surgeon.

The Dutch Surgical Society (NVvH, Nederlands Vereniging voor Heelkunde) and Amsterdam Skills Centre (ASC) identified the opportunity to offer interactive online education to Dutch surgical residents by livestreaming surgical procedures and technique on human cadavers in a nationwide initiative. The current surgical residency program contains a total of 488 residents, 135 first postgraduate year (PGY) residents-including urology, orthopedic, and plastic surgery residents in their general surgery rotation -, and 63-78 residents per PGY's 2-6. An online platform enabled interaction between surgeons, lecturers, and residents, thereby mimicking the interaction between surgeons and their residents during onsite live surgery. Five surgical key-procedures were chosen and supplemented by lectures of specialist faculty, the first of which aired on April 9th, 2020. This manuscript therefore describes and evaluates the achievements of last year and presents a roadmap to livestreaming surgical procedures and technique in resident education. By sharing our experiences, we aim to demonstrate the possibilities of livestreaming surgery for educational purposes, and to inspire others to adopt this promising digital resource in their curricula.

\section{Methods}

\section{Content development of livestreams}

To maintain surgical education, the $\mathrm{NVvH}$ consulted with the ASC, which is affiliated with the Amsterdam UMC, to discuss the feasibility of the livestreams in March 2020.
After the concept was deemed technically feasible, the boards of the NVvH sub-associations (on gastro-intestinal-, oncologic-, vascular-, pulmonary-, and trauma-surgery) were approached to discuss the execution of the livestreams and contents (Fig. 1). The NVvH prioritized residents in their first four postgraduate years (PGY's) as target audience for the first three livestreams. This, because a prolonged interruption of the education of these residents was particularly detrimental for their development and clinical activities $[2,5,6]$. To enable the highest attendance possible and to accommodate cooperating surgeons, the $\mathrm{NVvH}$ decided to broadcast all the livestreams after workhours at $5 \mathrm{pm}$. The livestreams duration was limited to $1.5-2 \mathrm{~h}$ to maintain optimal attention.

The boards of the respective sub-associations contacted their members to recruit faculty for the livestreams. These experts in their respective fields determined which surgical procedures were most appropriate based on the national surgical curriculum [7], and whether it was feasible within the timeframe defined by the $\mathrm{NVvH}$ - the actual livestreaming would have to be effected within four weeks. Therefore the laparoscopic cholecystectomy, intramedullary tibial fixation, lower leg amputation, total thyroidectomy, and introduction to pulmonary surgery were chosen.

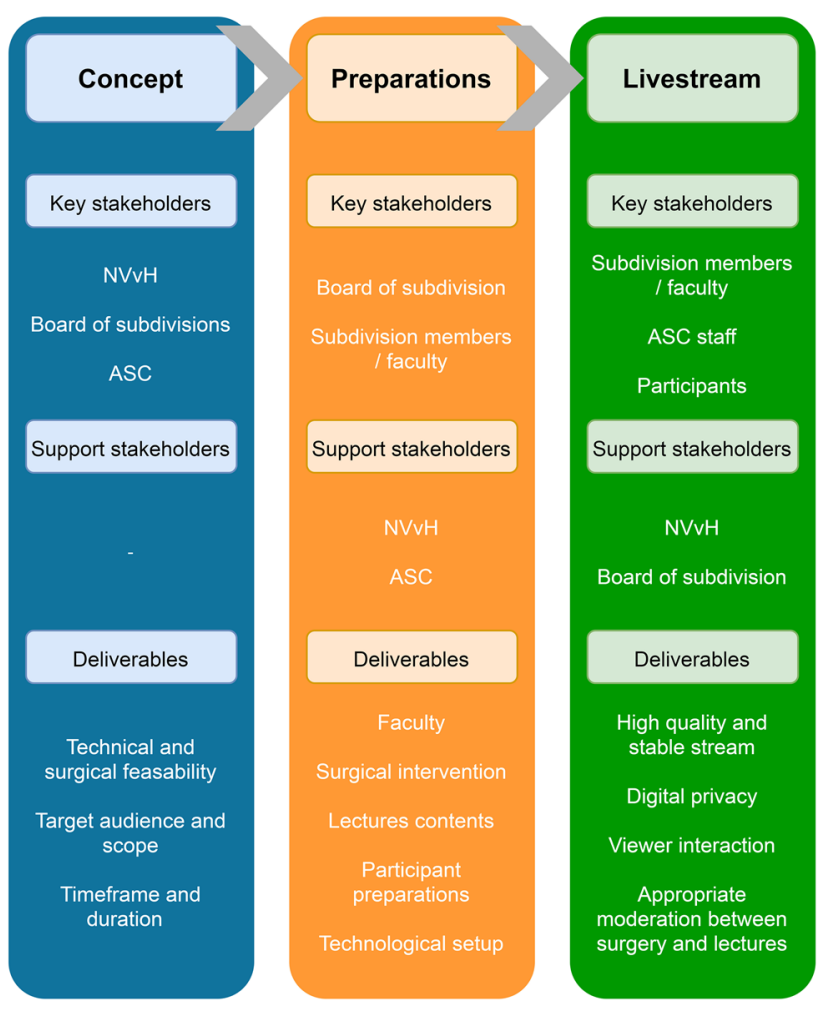

Fig. 1 Roadmap and summary of content development of surgical livestreams 
With the selected surgical procedures as backbone, the faculty decided on specific learning objectives for participants and accompanying explanatory topics. Etiology, symptoms, and diagnosis were covered, as were anatomy, surgical approach, pathology, and prognosis. Next, the performing surgeons and speakers were elected and the lectures were composed. Each livestream brought new experiences that were used to adapt contents, goals, timeframe, and script to support the faculty.

Based on the selected surgical procedure and learning objectives, the faculty of each livestream established what elements to cover in the participant preparation. A combination of existing e-learnings, available video materials, and relevant guidelines was selected and made digitally available to participants for self-study in the weeks before the livestream. Additionally, a voluntary pretest was made available for participants to test their knowledge before participating in the livestreams. All Dutch 1-4 years surgical residents were then approached by the $\mathrm{NVvH}$ and the Association of Surgical Residents (Vereniging van Assistent-Geneeskundigen in de Heelkunde, VAGH) to register for the livestream by following a link and creating a login on the secure online platform. The preparation for the livestream was distributed by the ASC to all the registered residents.

\section{Technological setup}

\section{Location and cadavers}

The ASC is an existing high-level training center containing a wetlab where live surgical procedures on human cadavers can be performed for training health care workers. PreCOVID this took place in a setting with faculty and trainees present at the operating tables. In accordance with national laws and regulations, all individuals voluntarily donated their body to science, and consent is obtained from all that their body will be used for scientific and educational purposes. In the Netherlands, such donations are made to anatomy departments of university medical centers. After death, the donated bodies are then transferred to the ASC. The ASC has its own ethical board containing the heads of the both anatomy departments of Amsterdam UMC, a professor in ethics, as well as several surgeons and legal experts who advice and decide on all matters concerning the ethical use of human tissue.

\section{Hardware and software}

A custom high-performance computer was used to incorporate audio and video signals from the wetlabs and lectures in the conference rooms. Video and images were incorporated in the livestream with Open Broadcaster Software ${ }^{\circledR}$, a free and open-source cross-platform streaming and recording program, and streamed using Vimeo (InterActiveCorp, New York City) streaming services. The Vimeo platform was chosen above others (such as Twitch and YouTube) because it offers unrestricted video availability to participants with a personal login, and allows the host to disable screen recording or easy downloading of the livestream. This functionality was essential to protect the privacy of the human cadavers, surgeons, and participants. Second, the platform was preferred above video conferencing services because it offers a dependable, high quality, and stable stream which incorporates videos, lectures, and images, and facilitates monitoring of these components. Furthermore, the platform provides a chat- and poll-function which were embedded into the livestream. Livestream hardware and software were extensively tested before the first stream, and constantly improved upon during the subsequent streams.

\section{The online learning platform}

Participants of courses in the ASC can request free access to online elements of the course through the ASC website, to prepare and augment their learning experience. Consequently, individuals without registration and permission cannot participate in courses or access videos and other leaning materials. All livestreams were broadcast within the learning platform environment that could be accessed on any device with an internet browser.

\section{Livestreaming support}

There was a viewing room next to the studio that was built in one of the conference rooms. Two ASC staff members monitored and adjusted video-, audio-, and livestream quality, supervised contents and viewer interaction, and were in charge of time management. Two additional ASC staff members were standby for extra support and troubleshooting between lab and studio, if necessary.

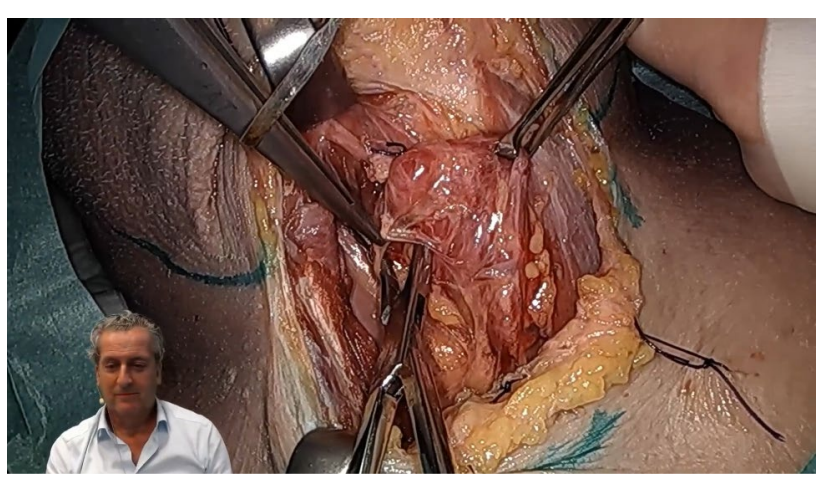

Fig. 2 Livestream image of right hemi thyroidectomy with picture-inpicture presenter (picture is placed with permission of presenter) 


\section{Viewer interaction}

The chat- and poll-function enabled interaction between participants, lecturers, and surgeons (Fig. 2). Designated faculty members moderated the input and output of these functions, while two ASC staff members provided technical and digital support. The designated faculty member(s) managed the questions and comments in the chat, and relayed them to the right person (i.e., the surgeon, lecture presenter) during the broadcast or answered the question themselves. Poll questions were devised in advance and integrated in the livestream at the appropriate timing.

\section{Surgery}

One surgeon and resident performed the procedure with a resident, assisted by two wetlab managers. Overview of video and audio was achieved through three cameras, two microphones, and an integrated speaker system. The surgeons explained their actions during the surgery, sometimes pausing to elaborate on technique or anatomy. The moderator of the livestream interacted with the surgeons, asking for clarification or to discuss the next course of action.

\section{Lectures}

One faculty member was designated as moderator of the livestream as a whole. They introduced the stream, faculty, and the lectures. The moderator or lecturers stood in front of a green screen when presenting, which enabled the simultaneous display of surgery and moderator using picture in picture. Figure 3 summarizes the technical setup of the livestreams and interaction flow of the livestreams.

\section{Analyses of livestream evaluations}

All participants were asked to voluntarily fill out a survey after each of the livestreams. Depending on the surgical procedure, the survey consisted of 19-23 questions and combined quantitative and qualitative items to enable a complete overview of the participants' experience. Surveys that were completed by surgical residents of PGY's 1-6 were
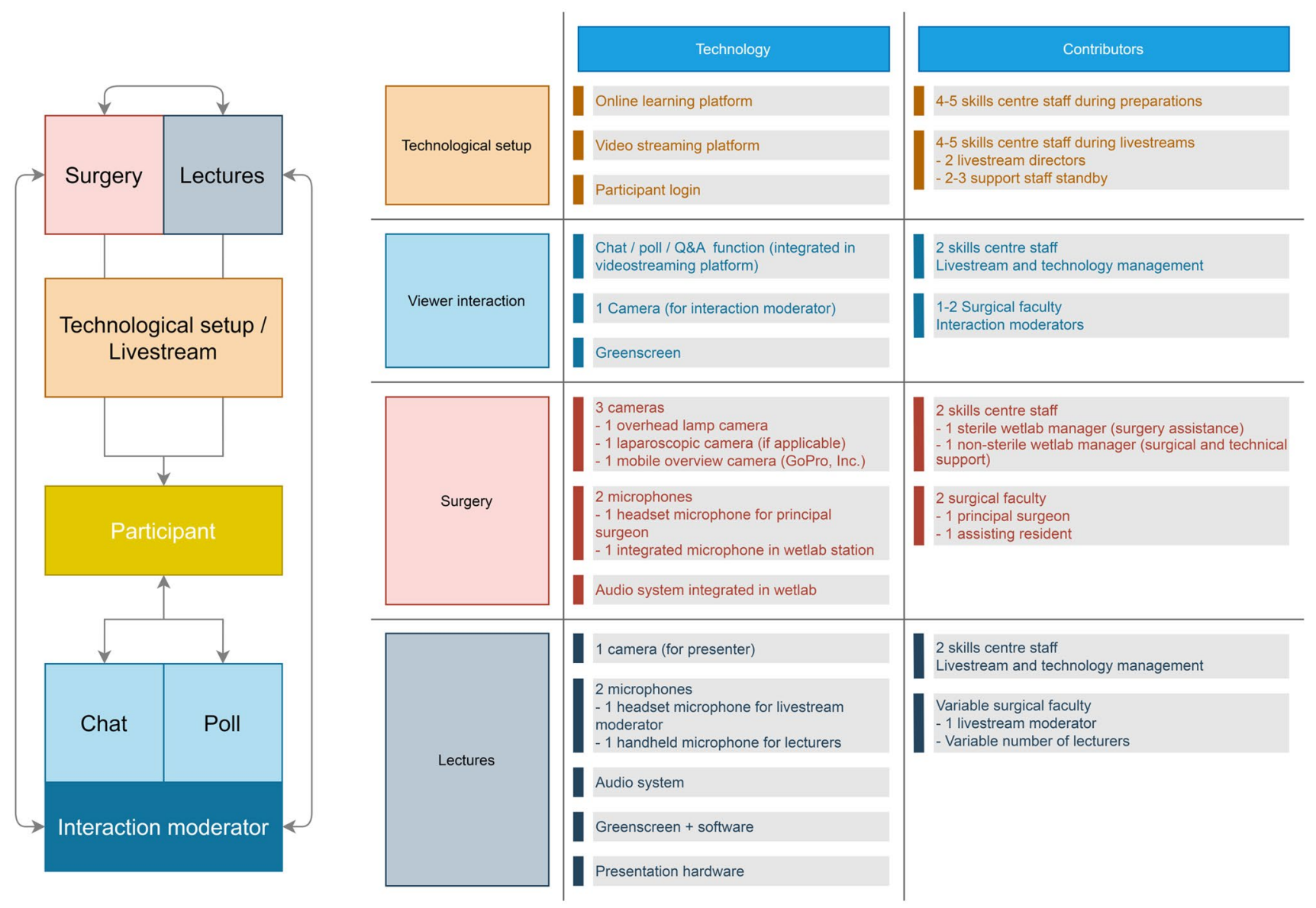

Fig. 3 Summary of livestream technology, setup, and flow of interactions during the livestream 
eligible for analysis. Descriptive analyses were performed on quantitative survey items using SPSS version 25.0 (IBM SPSS), analyses of qualitative results were performed using MaxQDA 2020 (VERBI Software GmbH). Approval of institutional review boards was not required because of the retrospective and observational character of this study.

\section{Results}

Each of the five livestreams attracted an audience ranging from 139 to 347 unique viewers. While the livestreams were created specifically for residents in training, also residents not in training and medical students viewed them as guests. Frequently, surgical staff allowed for group-viewing of the livestreams in lecture halls and staff rooms, while maintaining physical distance. The total reach of the livestreams is therefore not measurable, although it is certainly larger than the number of unique viewers recorded.

\section{Appreciation and recommendation}

A total number of 307 surveys were completed by participants, thereby corresponding with a response of $23-38 \%$ per livestream. Sixty percent $(n=185)$ were residents in training, $19 \%$ $(n=59)$ were medical students, $19 \%(n=59)$ had another function, did not report a function, or skipped most of the survey, and $1 \%(n=4)$ were residents not in training. The vast majority of residents in training rated and recommended the livestreams with at least a seven on a scale form 1-10 (Fig. 4), and the mean livestream rating and recommendation of the residents

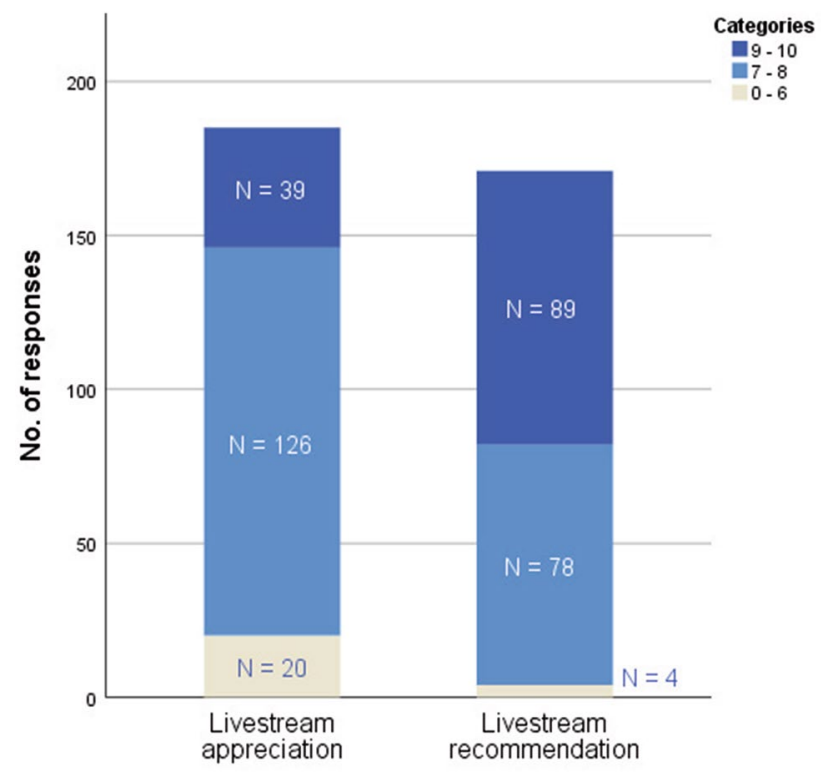

Fig. 4 Appreciation and recommendation of livestreams in absolute numbers in training were $7.7 \pm 1.1$ and $8.6 \pm 1.1$, respectively. There was little variety in these numbers based on postgraduate year (PGY) or livestream number (Fig. 5a, b), although the second livestream had the lowest appreciation due to technical issues.

Three out of four participants that gave a 6 or lower as livestream recommendation did so due to technical issues $(n=3)$ such as problems with logging in or an unstable connection-all in livestream two-while one resident suggested to a discussion covering the surgical technique and decision-making could have added value. Multiple residents, who scored 7 or 8 , commented that the theoretical discussion was too extensively at the expense of the surgery $(n=6)$, that a start time $5 \mathrm{pm}$ was too early $(n=5)$, and the difficulty level was too low for more senior residents. The group which graded the livestreams with a 9 or 10 did so because of the interaction between faculty and participants $(n=14)$, the overall educative value $(n=12)$, the combination of theory and practice $(n=9)$, the level of detail of the contents $(n=7)$, the performance of the faculty $(n=7)$, and the value of viewing a live surgery $(n=5)$. Several residents remarked that the offered interaction was of great value to the streams, both the interaction with the participants and the interaction between multiple surgeons. The first was appreciated for the engagement of the participants, while the latter was appreciated because a discussion between multiple surgeons from different hospitals seldom occurs and offered interesting points of view and different surgical techniques.

\section{Livestream components evaluation}

All components of the livestreams were predominantly rated as positive to strongly positive on a five point Likert-scale, with 'use of moderators' and 'balance between theory and practice' rated best (Fig. 6). In the qualitative analysis, residents specifically appreciated the possibility to follow the interaction between multiple surgeons and hear their different surgical approaches and techniques during surgery. Additionally, residents agreed that the theory should be used as background and be in support of the surgery, without overruling the practical execution. Time of day received the most negative and neutral ratings; in the qualitative analysis residents agreed that starting at $5 \mathrm{pm}$ interfered with clinical duties.

Low ratings of livestreams were given because of technical issues, which especially interrupted the second livestream. Several participants were unable to watch the livestream midway onward due to a connectivity outage followed by problems logging in for a second time. Additionally, sound was delayed or absent during parts of streams two and four for some participants. 
Fig. 5 a and b Mean appreciation and recommendation per livestreams per PGY (postgraduate year)

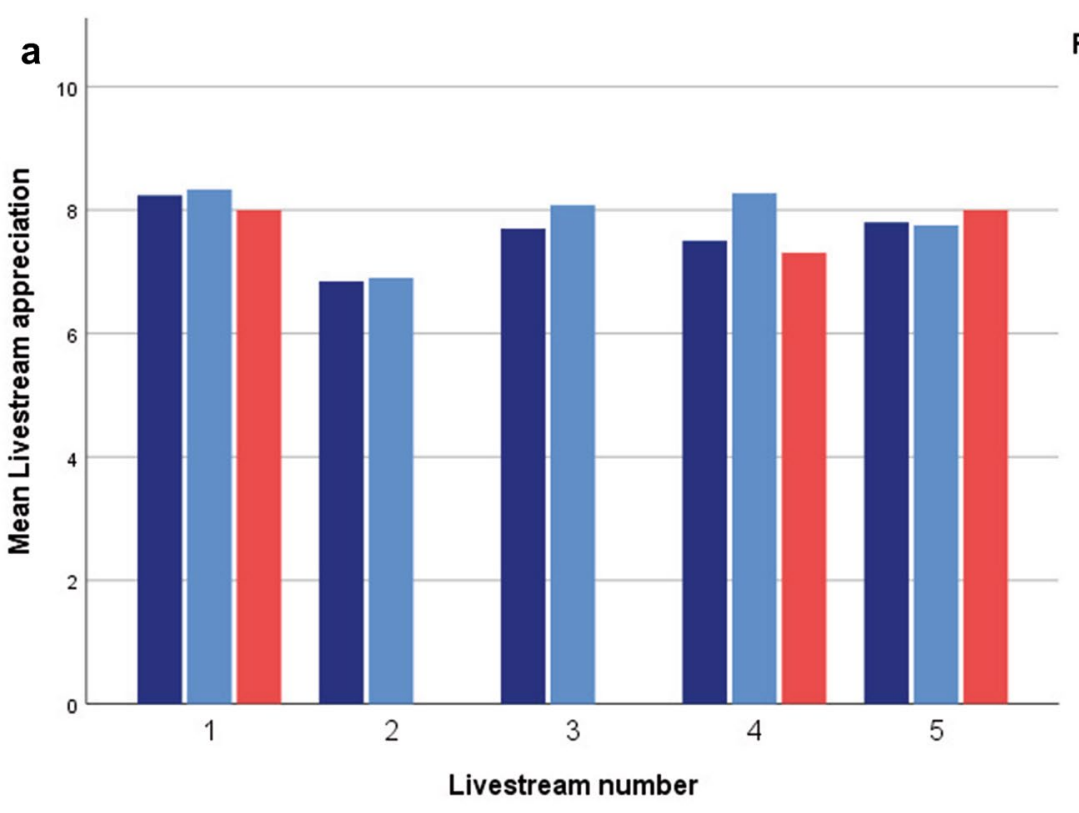

Postgraduate year (PGY)

DGY 1 \& 2

$P G Y 3 \& 4$

DGY 5 \& 6

\section{b}

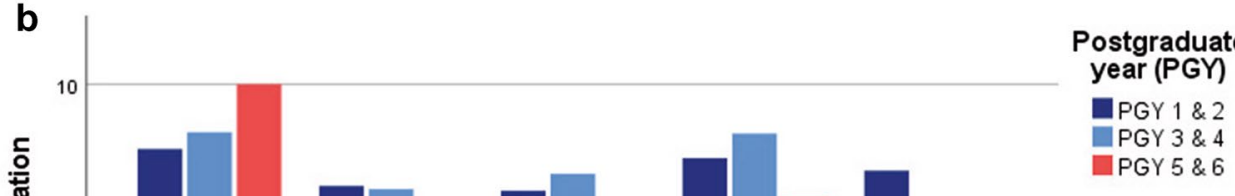

\section{Discussion}

Livestreaming surgical procedure and technique on human cadavers in our nationwide initiative has proven to be useful, feasible, and very well appreciated by surgical residents. Although use of video in surgical education cannot substitute the learning experience of performing or assisting in real-life surgery, live video streaming does provide the experience of blended learning through the combination of surgical theory with practice, while enabling a two-way interaction between the audience and the performing surgical specialists. Easy access and a stable stream are obviously essential for a successful livestream. Time of day, duration of the livestream, and difficulty level of livestream content are important factors to keep in mind, and should be adjusted to the target audience.

Our large-scale evaluation of participants validates the positive livestreaming experiences as previously referred to in literature. Use of live video streaming for demonstration of surgical technique is a feasible and valuable addition to the surgical education palette according to both surgeons and residents, especially during the COVID pandemic [8-10]. There are several differences between aforementioned studies in literature and our study. First of all, we performed surgical procedures on human cadavers as opposed to the surgical procedures on patients as published by Jack et al., Faiz et al., and Yuen et al. [8-10]. Because previous authors all presented data from the USA, legislation on digital privacy 


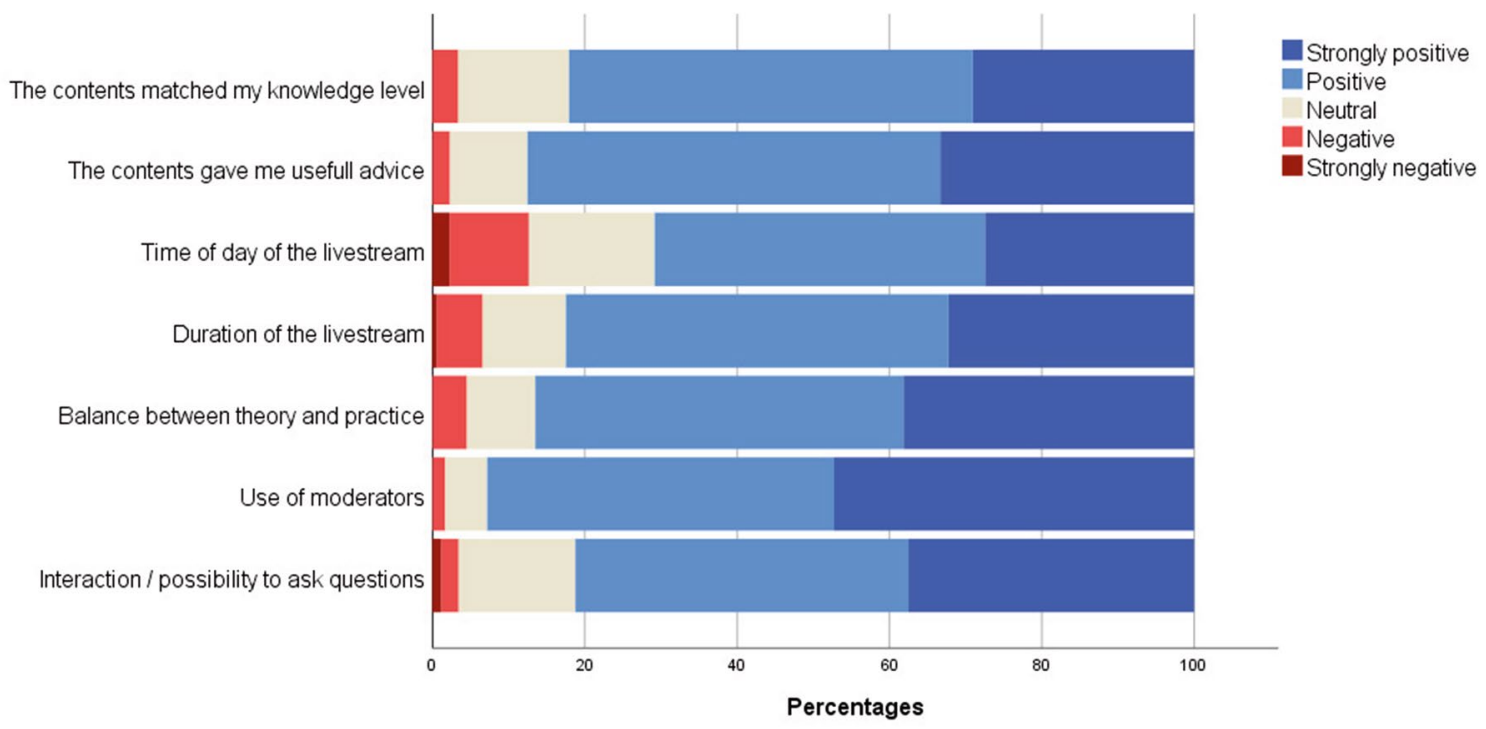

Fig. 6 Evaluation of livestream components on a five point Likert-scale

and security is different. There is no globally accepted set of laws or regulation that apply to digitally livestreaming surgical procedures, especially not with regard to streams of cadaver surgery. Two authors examining the question of (digitally) sharing anatomical images agree that there is little guidance from in the literature regarding ethics and safety, although there is an increasing risk of unethical or misuse $[11,12]$. In the ASC such an ethical board advices and decides on these matters, although (inter)national guidelines can support clinicians and educators now that education is being digitized. There are some key considerations to take into account which apply to both surgical procedures on patients and human cadavers. As shown in Table 1, perioperative precautions are necessary to assure the anonymity of the patient or cadaver, while digital measures are essential to protect privacy of the patient/cadaver and faculty. While cadaver surgery is valuable as hands-on training form, we believe that there are also distinct advantages to livestreaming cadaver surgery over surgery on patients; less stress and cognitive load of the surgeon, opportunity for a more elaborate interaction between multiple surgeons and residents, expanding the surgery and increasing surgical time and time for educational purposes, and legal and ethical considerations with regard to possible postoperative complications. Especially during this COVID pandemic, livestreaming for educational purposes from this setting has the great advantage over live surgery on patients of not further compromising scarce OR resources in the clinical COVID setting, nor jeopardizing the safety in the hospital setting by bringing in more people than strictly necessary for patient care. Additionally; COVID might interfere with planned livestream surgery on patients. Bringing live streaming surgery out of the hospital therefore safeguards both clinical setting and education.

There are myriad options to watch and learn from online surgical videos-such as WebSurg, AIS, CSurgeries, MedTube, GIBLIB, and YouTube-which are clearly appreciated by trainees $[4,13]$. There is little research to the quality of the videos, although most dedicated surgical education sites offer some kind of quality assurance, accreditation or peer review of uploaded videos-as opposed to Youtube ${ }^{\circledR}$ which is completely open source. Three studies assessed the reliability, quality, accuracy, and satisfaction with regard to videos of the critical view of safety, inguinal hernia repair, and sleeve gastrectomies on Youtube ${ }^{\circledR}$, Websurg ${ }^{\circledR}$, and GIBLIB ${ }^{\circledR}$ [14-16]. All authors agree that

Table 1 Privacy and security components of livestreaming surgery

\begin{tabular}{ll}
\hline Medical and surgical factors & Digital factors \\
\hline $\begin{array}{l}\text { Written consent to both the surgery and the livestream (in case of a live sur- } \\
\text { gery) }\end{array}$ & $\begin{array}{c}\text { Secure digital livestreaming environment (according to local } \\
\text { laws and regulations) } \\
\text { Login protected access to the digital livestreaming environment } \\
\text { Appropriate pre-operative cover-up of the patient/specimen }\end{array}$ \\
$\begin{array}{l}\text { No disclosure of patient/specimen specific data during the livestream and lives- } \\
\text { tream suspension during pre- and postoperative time-out verification }\end{array}$ & $\begin{array}{l}\text { Disable downloading of the livestream } \\
\end{array}$
\end{tabular}


while the opportunity and satisfaction these videos offer is significant, their quality is often less than appropriate-even the videos which were reviewed and prepared by clinicians. Especially information with regard to pre- and postoperative processes is often limited, and there is no significant superiority of GIBLIB ${ }^{\circledR}$ and WebSurg ${ }^{\circledR}$ over YouTube ${ }^{\circledR}$. With the exception of Giblib, the aforementioned sites are free to use, although with the exception of Websurg and Csurgeries all others require an account to get access to the videos. While these videos offer a wide range of surgical procedures, they lack the live immersive interaction which differentiates livestreaming surgery. Residents indicated that this interaction was highly appreciated, and we believe that this interaction is essential to align the contents of surgical videos to the knowledge and questions of the audience, and transforms a passive movie-watching occasion into an active learning experience. Combining prerecorded videos with a live discussion could be a possible alternative. An advantage this application would be lower costs and a wider applicability without the need for advanced technology. To best mimic the interaction with the performing surgeon like in cadaver surgery, preferably the surgeon or surgical team that recorded the video takes part in the discussion. However, zooming in on a certain part of the procedure, literally or by expanding the surgery or surgical time- one of the key advantages of cadaver surgeries in our study-is not possible in this application of surgical videos.

We and previous authors did not measure the impact of livestreams on the clinical performance of residents and efficacy, which limits our study and the available evidence [8-10]. Future studies should focus on the quantitative evaluation of the effects of video based resources and livestreams on resident performance. However, it should be taken into account that these resources are complementary to the indispensable hands-on training — not a replacement-and should be researched and evaluated accordingly. Moreover, a problem when evaluating complementary training resources such as videos and livestreams, especially in a nationwide setting, is the variability in previous exposure of residents, and the unworkability of withholding residents from further training during a trial. This makes quantitative evaluation in for instance a randomized trial nearly impossible and introduces bias and confounders in practically all types of quantitative research. Another limitation is the response rate to the surveys of only $23-38 \%$. Also, we did not collect information whether the respondents were new participants or had joined a previous livestream, partly because of privacy regulations. This may have impacted the response rates of the surveys. Lastly, the surgeries performed were quite heterogeneous. The cadaveric model may be more or less effective for the given procedure. We did not evaluate this specific aspect. Future evaluations should focus on learning effects among different procedures.
The impact on clinical performance needs to be quantified to consolidate the role of livestreams in surgical education, and should therefore be subjected to future studies. The COVID-19 pandemic has made clear how vulnerable surgical resident training is due to the dependability on surgical caseload $[2,17]$. Digital training resources such as livestreams, virtual/augmented/mixed reality, and simulators may become indispensable in providing sufficient surgical exposure to residents-although they will never replace the need for hands-on operating room experience. By combining the best of both the physical and digital world in blended learning, hybrid curricula can be established to future proof surgical education and to train proficient future surgeons.

\section{Conclusion}

Livestreaming of surgical procedures proved a feasible and very well appreciated augmentation of resident education during the COVID pandemic. In times of social distancing and limited surgical caseload, interactive, immersive livestreaming added value to traditional surgical training. Digital resources show great promise in providing blended learning environments that allow for sufficient learning experiences for residents that do not depend on clinical exposure.

Acknowledgements The authors declare that the following persons are co-authors who participated through the NVvH livestream collaboration group on this study and accept direct responsibility for the manuscript:

Jerry Middelberg, Ilona Veenema, Goos Huijzer, KlaasWalter Meyer, KerriLee Colman, Esther Jacobs, Olivier R. C. Busch, Jeroen Hagendoorn, Rutger-Jan Swijnenburg, Hilko A. Swank, J. Sven D. Mieog, Bas Groot Koerkamp, Michiel H. J. Verhofstad, Johan G. H. van den Brand, Martin J. Heetveld, Floortje Huizing, Sven A. G. Meylaerts, Jan-Willem H. P. Lardenoije, Jaap F. Hamming, Joris J. Blok, Marlies J. Keijzers, Jan Willem H. C. Daemen, Schelto Kruijff, Tessa M. van Ginhoven, Lutske Lodewijk, Yvonne L. J. Vissers, Geertruid Marres, Koen J. Hartemink, Wout B. Barendregt, J. Wolter A. Oosterhuis, Merlijn Hutteman, Donald van der Peet

Affiliations

Jerry Middelberg, Ilona Veenema, Goos Huijzer, Klaas Walter Meyer, Kerri Lee Colman: Amsterdam Skills Centre (ASC), Amsterdam, The Netherlands

Esther Jacobs: Nederlandse Vereniging voor Heelkunde $(\mathrm{NVvH})$, Domus Medica, Utrecht, The Netherlands

Olivier R.C. Busch, Rutger-Jan Swijnenburg, Hilko A. Swank, Donald van der Peet: Department of Surgery, Amsterdam Gastroenterology and Metabolism, Amsterdam UMC, University of Amsterdam, The Netherlands

Jeroen Hagendoorn: Department of Surgery, University Medical Centre Utrecht, Utrecht, The Netherlands

J. Sven D. Mieog, Jaap F. Hamming, Joris J. Blok: Department of Surgery, Leiden University Medical Center, Leiden, The Netherlands. Merlijn Hutteman: Department of Surgery \& department of Cardiothoracic Surgery, Leiden University Medical Center, Leiden, The Netherlands,

Bas Groot Koerkamp, Tessa M. van Ginhoven: Department of Surgery, Erasmus University Medical Centre, Rotterdam, The Netherlands 
Michiel H.J. Verhofstad: Trauma Research Unit, Department of Surgery, Erasmus MC, University Medical Center Rotterdam, PObox 2040, 3000 CA Rotterdam, The Netherlands

Johan G.H. van den Brand: Department of Surgery, Northwest Clinics, Alkmaar, The Netherlands

Martin J. Heetveld: Department of Surgery, Spaarne Gasthuis, Haarlem, The Netherlands

Floortje Huizing, Sven A.G. Meylaerts, J. Wolter A. Oosterhuis: Department of Surgery, Haaglanden Medical Centre, Den Haag, The Netherlands

Jan-Willem H.P. Lardenoije: Department of Sugery, Rijnstate Hospital, Arnhem, The Netherlands

Marlies J. Keijzers, Jan Willem H.C. Daemen: Department of Surgery, Maastricht University Medical Centre, Maastricht, The Netherlands

Schelto Kruijff: Department of Surgery, University Medical Center Groningen, The Netherlands

Lutske Lodewijk: Department of Surgery, Diakonessen Hospital, Utrecht, The Netherlands

Yvonne L.J. Vissers: Department of Surgery, Zuyderland Medical Center, Heerlen, The Netherlands

Geertruid Marres: Department of Surgery, Albert Schweitzer Hospital, Dordrecht, The Netherlands

Koen J. Hartemink: Department Surgery, the Netherlands Cancer Institute-Antoni van Leeuwenhoek Hospital, Amsterdam, The Netherlands

Wout B. Barendregt: Department of Surgery, Canisius-Wilhelmina Ziekenhuis, Nijmegen, The Netherlands

\section{Declarations}

Disclosures Tim M. Feenstra, L. Peter van Felius, Menno R. Vriens, Laurents P.S. Stassen, Gijs J.D. van Acker, Els J.M. Nieveen van Dijkum, Marlies P. Schijven, H. Jaap Bonjer, Jerry Middelberg, Ilona Veenema, Goos Huijzer, Klaas Walter Meyer, Kerri Lee Colman, Esther Jacobs, Olivier R.C. Busch, Jeroen Hagendoorn, Rutger-Jan Swijnenburg, Hilko A. Swank, J. Sven D. Mieog, Bas Groot Koerkamp, Michiel H.J. Verhofstad, Johan G.H. van den Brand, Martin J. Heetveld, Floortje Huizing, Sven A.G. Meylaerts, Jan-Willem H.P. Lardenoije, Jaap F. Hamming, Joris J. Blok, Marlies J. Keijzers, Jan Willem H.C. Daemen, Schelto Kruijff, Tessa M. van Ginhoven, Lutske Lodewijk, Yvonne L.J. Vissers, Geertruid Marres, Koen J. Hartemink, Wout B. Barendregt, J. Wolter A. Oosterhuis, Merlijn Hutteman, and Donald van der Peet have no conflict of interest or financial ties to disclose.

\section{References}

1. Francis N, Dort J, Cho E et al (2020) SAGES and EAES recommendations for minimally invasive surgery during COVID-19 pandemic. Surg Endosc 34(6):2327-2331

2. Kapila AK, Farid Y, Kapila V, Schettino M, Vanhoeij M, Hamdi M (2020) The perspective of surgical residents on current and future training in light of the COVID-19 pandemic. Br J Surg 107:e305
3. Tinelli G, Sica S, Minelli F, Tshomba Y (2020) Vascular surgery education during COVID-19 pandemic. J Vasc Surg 72(2):763-764

4. Mahendran B, Celentano V, Soltes M et al (2020) EAES online educational resources: a survey of the membership of the European Association for Endoscopic Surgery (EAES). Surg Endosc 35:2059

5. Coe TM, Jogerst KM, Sell NM et al (2020) Practical techniques to adapt surgical resident education to the COVID-19 era. Ann Surg 272(2):e139-e141

6. Dedeilia A, Sotiropoulos MG, Hanrahan JG, Janga D, Dedeilias P, Sideris M (2020) Medical and surgical education challenges and innovations in the COVID-19 era: a systematic review. In Vivo 34(3 Suppl):1603-1611

7. (NVvH) NVvH (2020) Opleidingsplan heelkunde: structuur curriculum heelkunde voor reflectieve professionals (SCHERP) 2.1

8. Jack MM, Gattozzi DA, Camarata PJ, Shah KJ (2020) Livestreaming surgery for medical student education-educational solutions in neurosurgery during the COVID-19 pandemic. J Surg Educ 78:99

9. Yuen JC, Gonzalez SR, Osborn T, Abu-Rmaileh M (2020) Untethered and HIPAA-compliant Interactive Livestreaming of Surgery to Residents and Medical Students. Plast Reconstr Surg Glob Open 8(10):e3165

10. Faiz T, Marar O, Kamel MK, Vance S (2020) Teaching Operative Surgery to Medical Students Using Live Streaming During COVID-19 Pandemic. Surg Innov 28:253

11. Cornwall J, Callahan D, Wee R (2016) Ethical issues surrounding the use of images from donated cadavers in the anatomical sciences. Clin Anat 29(1):30-36

12. Hennessy CM, Royer DF, Meyer AJ, Smith CF (2020) Social media guidelines for anatomists. Anat Sci Educ 13(4):527-539

13. Celentano V, Smart N, Cahill RA et al (2019) Use of laparoscopic videos amongst surgical trainees in the United Kingdom. Surgeon 17(6):334-339

14. Chavira AM, Rivas JF, Molina A et al (2021) The educational quality of the critical view of safety in videos on youtube $(\mathrm{R})$ versus specialized platforms: which is better? Critical view of safety in virtual resources. Surg Endosc 2021:1-9

15. Ferhatoglu MF, Kartal A, Filiz AI, Kebudi A (2019) Comparison of new era's education platforms, YouTube(R) and WebSurg(R), in sleeve gastrectomy. Obes Surg 29(11):3472-3477

16. Kartal A, Kebudi A (2019) Evaluation of the reliability, utility, and quality of information used in total extraperitoneal procedure for inguinal hernia repair videos shared on WebSurg. Cureus 11(9):e5566

17. Porpiglia F, Checcucci E, Amparore D et al (2020) Slowdown of urology residents' learning curve during the COVID-19 emergency. BJU Int 125(6):E15-E17

Publisher's Note Springer Nature remains neutral with regard to jurisdictional claims in published maps and institutional affiliations. 